\title{
Non-small-cell Carcinoma Localised within the Pulmonary Artery Misdiagnosed as Pulmonary Thromboembolism
}

\author{
Zeyad Albadri ${ }^{1}$, Omar Khattab ${ }^{2}$ \\ ${ }^{1}$ Rosenlund Medical Centers, Jönköping, Sweden \\ ${ }^{2}$ Ryhov University Hospital, Radiology Department, Jönköping, Sweden
}

Received: 22/09/2019

Accepted: 23/09/2019

Published: 08/11/2019

How to cite this article: Albadri Z, Khattab O. Non-small-cell-carcinoma localised with the pulmonary artery misdiagnosed as pulmonary thromboembolism. EJCRIM 2019;6: doi:10.12890/2019_001284.

Conflicts of Interests: The Authors declare that there are no competing interest

This article is licensed under a Commons Attribution Non-Commercial 4.0 License

\section{ABSTRACT}

In this case report we describe a 69-year-old male ex-smoker with non-small-cell carcinoma localised within the pulmonary artery, misdiagnosed as pulmonary thromboembolism. This case indicates that non-small-cell carcinoma can be localised within the pulmonary artery. Furthermore, it emphasises the importance of performing a positron emission tomography scan and diagnostic intraluminal biopsy in unexplained lesions in the pulmonary artery to reach the proper diagnosis at the early stages of the disease. The patient is currently undergoing concurrent chemotherapy and radiation therapy.

\section{LEARNING POINTS}

- Tumours within the pulmonary arteries are a rare finding and rarely reported, and are often misdiagnosed as pulmonary thromboembolism.

- If the diagnosis is unclear a positron emission tomography (PET-CT) scan and intravascular biopsy should be considered at early stages of the presentation.

- In general, surgery would be the treatment of choice for pulmonary artery tumours but this is not optimal and treatment options remain controversial.

\section{KEYWORDS}

Lung cancer, intravascular tumour, pulmonary thromboembolism

\section{CASE DESCRIPTION}

A 69-year-old male ex-smoker presented to the emergency department with acute-onset right-sided chest pain with dyspnoea. His past medical history included chronic obstructive pulmonary disease stadium-3, well controlled with medications. On presentation, his oxygen saturation was 93\%. Physical examination of the patient showed no abnormalities. Laboratory findings showed a normal full blood count, normal venous blood gas and C-reactive protein levels, and no evidence of clotting disorders. Conventional chest radiography showed no abnormal shadows. The patient was admitted to our hospital for further management. On Day 2 of admission the patient's general condition deteriorated. A computed tomography (CT) chest angiogram was performed which revealed a $5.7 \times 4.2 \times 5.0 \mathrm{~cm}$ mass in the right pulmonary artery; no enlarged lymph nodes or signs of metastasis were found (Fig. 1).

After discussion with several radiologists, a decision was made to manage the patient as a case of thromboembolism occlusion in the right pulmonary artery. He received appropriate anticoagulation therapy, but no improvement occurred. A follow-up CT chest angiogram was carried out 2 weeks later, which showed no improvement; however, a new finding of $1.5 \mathrm{~cm}$ pleural fluid in the right lung was discovered (Fig. 2). 

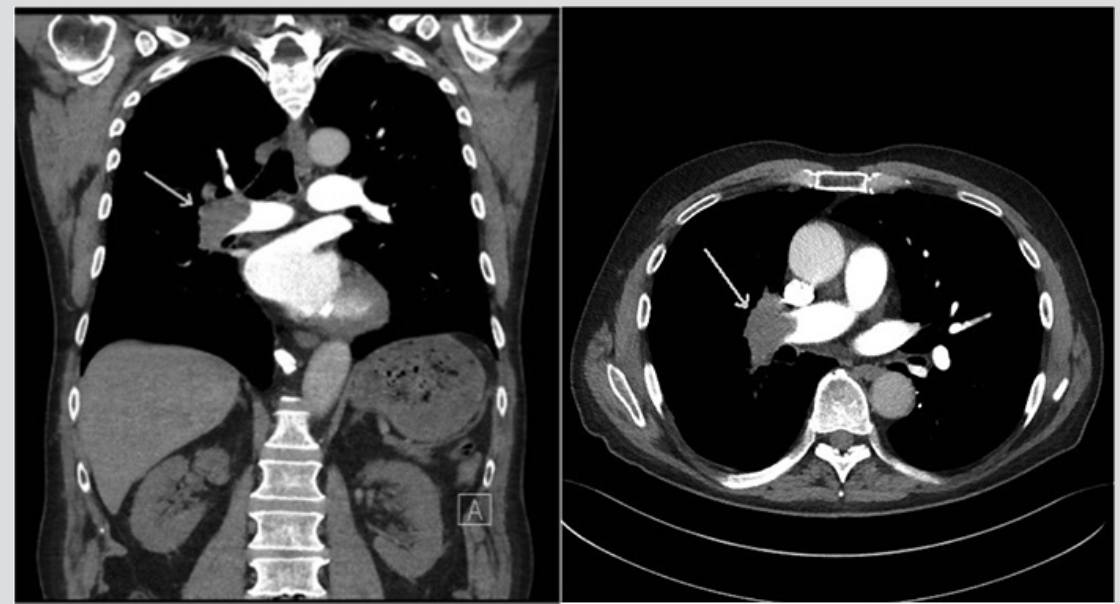

Figure 1. Computed tomography (CT) chest angiogram 2 days after presentation showing a mass within the right main pulmonary artery (arrows).

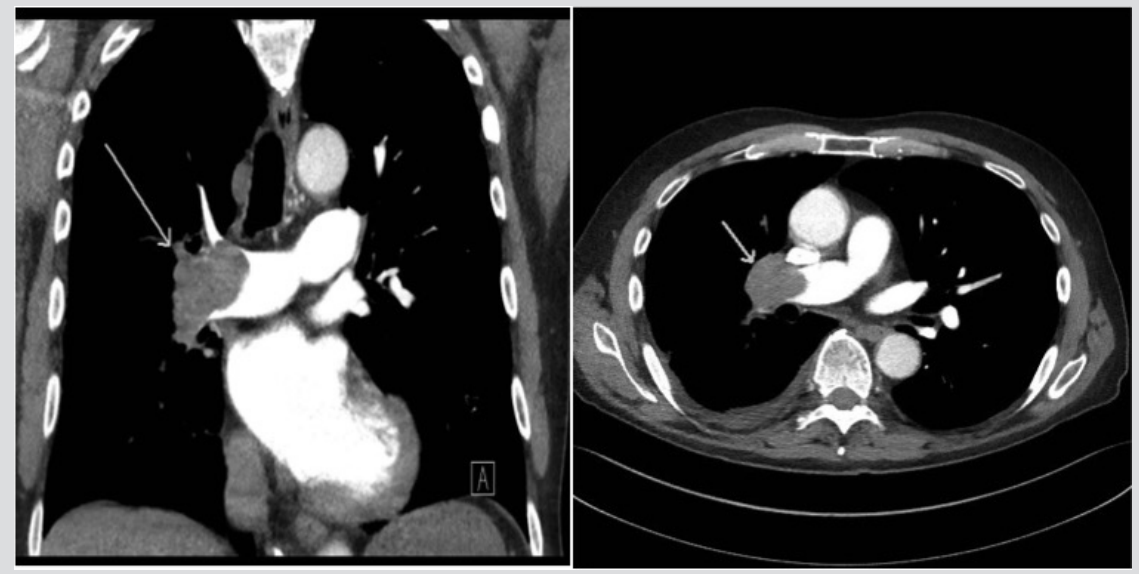

Figure 2. Computed tomography (CT) chest angiogram 2 weeks later showing no improvement in the mass localised within the right pulmonary artery (arrows).

A decision was made to proceed further with magnetic resonance imaging (MRI) of the chest which revealed increasing pleural fluid with intraluminal filling defects involving the right pulmonary artery, citing pulmonary thromboembolism as the main differential diagnosis (Fig. 3). A PET-CT scan was performed after a multidisciplinary conference, which revealed a large invasive obstructing homogenous mass in the right pulmonary artery with high fluorodeoxyglucose (FDG) uptake of 25.2 SUV max, no FDG uptake in mediastinal lymph nodes or other organs; the differential diagnoses included pulmonary thromboembolism and an intrapulmonary artery tumour (Fig. 4). The patient underwent endoscopy-guided biopsy of the mass and histopathology was consistent with the diagnosis of non-small-cell carcinoma. The patient is currently receiving ongoing chemotherapy and radiotherapy.

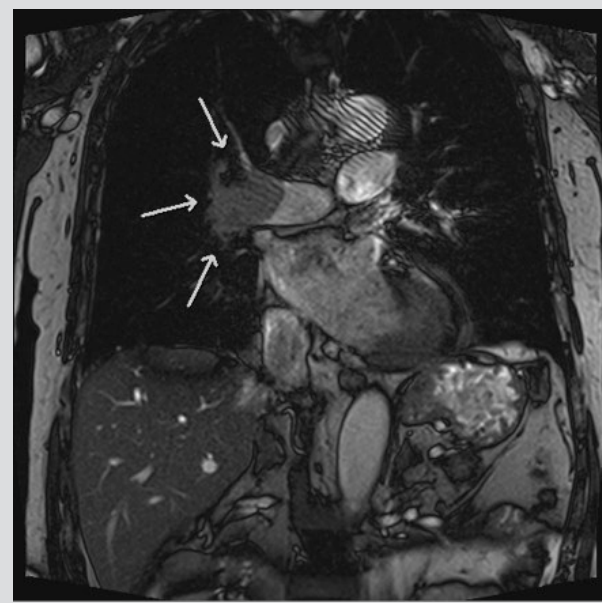

Figure 3. Magnetic resonance imaging (MRI) chest scan showing multiple filling defects in the right main pulmonary artery (arrows). 


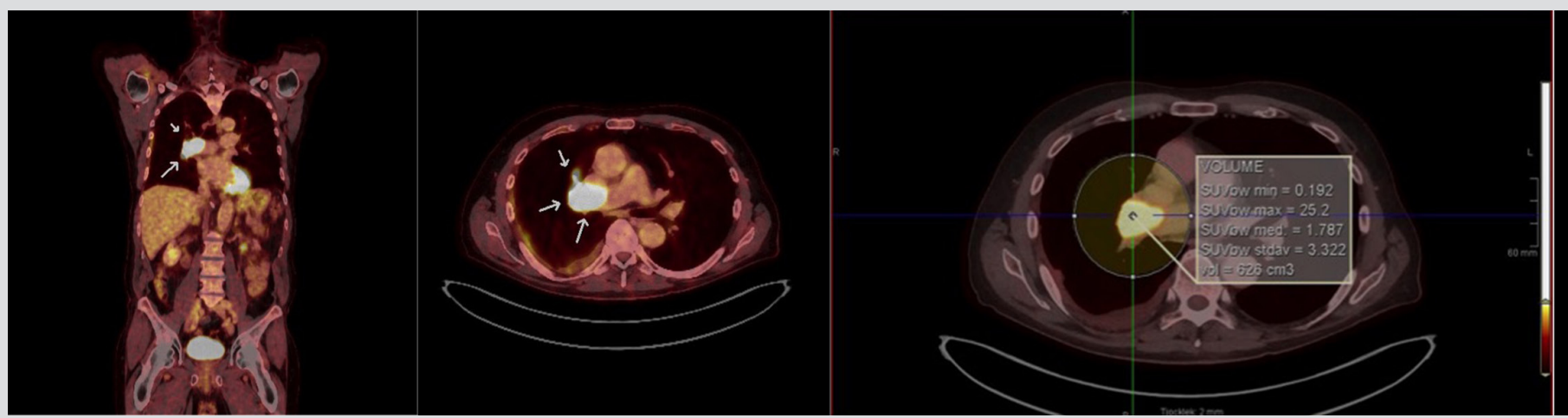

Figure 4. Positron emission tomography (PET-CT) scan showing fluorodeoxyglucose (FDG) uptake in the mass in the right main pulmonary artery (arrows) with precise FDG uptake measurements.

\section{DISCUSSION}

We experienced a case of a man with non-small-cell carcinoma which was exclusively localised within the right pulmonary artery. To the best of our knowledge, no similar cases have been reported in the literature previously. The pulmonary vessels may be involved by different primary and secondary tumours. The primary aetiologies of masses existing in the intrapulmonary artery include angiosarcoma, pulmonary thromboembolism, tumour embolisation from cancers of other organs and invasion of a primary lung cancer into the intravascular lumen ${ }^{[1-8]}$. There are very few reports describing intravascular tumours within the pulmonary arteries in the literature ${ }^{[6]}$.

In our case, the patient presented with acute-onset chest pain and the first CT chest angiogram showed a filling defect in the right pulmonary artery. The radiologists were divided into two groups, a group that suspected that the radiological findings were consistent with pulmonary thromboembolism and another group that completely disagreed with this and suspected the findings to be more sinister. The patient's symptoms did not improve and a follow-up CT chest angiogram showed no change in the filling defect despite appropriate anticoagulation therapy. The turning point in this case was when the patient underwent a PET-CT scan which showed malignant features with a differential diagnosis of sarcoma. We found no evidence of metastasis from any other organs.

Previous case reports have shown that clinical and imaging manifestations of intrapulmonary tumours may mimic those of pulmonary embolism, and therefore, reaching a diagnosis before biopsy or autopsy is difficult ${ }^{[1,4,8]}$. Dyspnoea, chest pain, cough and haemoptysis are the most common presenting symptoms in both conditions. Furthermore, both of the diseases initially appear as intraluminal filling defects in the pulmonary artery systems on contrast-enhanced CT scans. It is very important to differentiate between the diseases to avoid misdiagnosis of potentially fatal malignancy and unnecessary anticoagulation therapy.

In the present case, PET-CT scanning provided crucial information for diagnosis, since the diagnostic biopsy was performed based on the abnormal findings from the PET-CT scan. A study in 2013 reported that PET-CT scans may be beneficial in making a differential diagnosis between primary intrapulmonary tumours and pulmonary thromboembolism ${ }^{[9]}$. There have been previous case reports of metastasis or direct invasion to the pulmonary artery from other organs ${ }^{[10]}$. However, in this case, we found no systemic or imaging findings that indicated the presence of malignancy in other organs.

Our conclusion is that non-small-cell carcinoma can be confined within the pulmonary arteries and that clinicians should maintain high clinical suspicion of intraluminal primary tumours in patients with a filling defect in the pulmonary artery without any underlying risk factors for pulmonary thromboembolism and worsening of the symptoms despite appropriate anticoagulation therapy. 


\section{REFERENCES}

1. Brecher CW, Lang EV. Tumor thromboembolism masquerading as bland pulmonary embolism. J Vasc Interv Radiol 2004;15:293-296.

2. Kayalar N, Leibovich BC, Orszulak TA, Schaff HV, Sundt TM, Daly RC, et al. Concomitant surgery for renal neoplasm with pulmonary tumor embolism. J Thorac Cardiovasc Surg 2010;139:320-325.

3. Parish JM, Rosenow III EC, Swensen SJ, Crotty TB. Pulmonary artery sarcoma: clinical features. Chest 1996;110:1480-1488.

4. Zurick III AO, Lenge De Rosen V, Tan CD, Rodriguez ER, Flamm SD, Schoenhagen P. Pulmonary artery intimal sarcoma masquerading as pulmonary embolism. Circulation 2011;124:1180-1181.

5. Estrera AL, Cagle PT, Azizzadeh A, Reardon MJ. Large cell neuroendocrine carcinoma: an unusual presentation. Ann Thorac Surg 2002;73:1957-1960.

6. Kamigaki M, Yamazaki K, Tsujino I, Suga M, Sakaue S, Dosaka-Akita H, et al. Small cell carcinoma of the lung exclusively localized within the left descending pulmonary artery. Chest 2005;127:2273-2276.

7. Yamaguchi T, Suzuki K, Asamura H, Kondo H, Niki T, Yamada T, et al. Lung carcinoma with polypoid growth in the main pulmonary artery: report of two cases. Jpn J Clin Oncol 2000;30:358-361.

8. Engelke C, Riedel M, Rummeny EJ, Marten K. Pulmonary haemangiosarcoma with main pulmonary artery thrombosis imitating subacute pulmonary embolism with infarction. Br J Radiol 2004;77:623-625.

9. Lee EJ, Moon SH, Choi JY, Lee KS, Choi YS, Choe YS, et al. Usefulness of fluorodeoxyglucose positron emission tomography in malignancy of pulmonary artery mimicking pulmonary embolism. ANZ J Surg 2013;83:342-347.

10. Goto T, Maeshima A, Kato R. Lung adenocarcinoma with peculiar growth to the pulmonary artery and thrombus formation: report of a case. World J Surg Oncol 2012;10:16. 